\title{
Dynamic OCT Signal Loss for Determining RPE Radiant Exposure Damage Thresholds in Microsecond Laser Microsurgery
}

\author{
Christian Burri ${ }^{1,2, *} \mathbb{1}$, Alessa Hutfilz ${ }^{3}$, Lorenz Grimm ${ }^{1}$, Simon Salzmann ${ }^{1}\left(\mathbb{D}\right.$, Patrik Arnold ${ }^{1}$, Boris Považay ${ }^{1}{ }^{1}$, \\ Christoph Meier ${ }^{1}$, Andreas Ebneter ${ }^{4} \mathbb{D}$, Dirk Theisen-Kunde ${ }^{3}$ and Ralf Brinkmann ${ }^{3,5} \mathbb{C}$
}

1 Institute for Human Centered Engineering (HuCE)-optoLab, Bern University of Applied Sciences, Quellgasse 21, 2501 Biel, Switzerland; lorenzsebastian.grimm@bfh.ch (L.G.); simon.salzmann@bfh.ch (S.S.); patrik.arnold@bfh.ch (P.A.); boris.povazay@bfh.ch (B.P.); christoph.meier@bfh.ch (C.M.)

2 Biomedical Photonics Group, Institute of Applied Physics (IAP), University of Bern, Sidlerstrasse 5, 3012 Bern, Switzerland

3 Medical Laser Center Lübeck (MLL), Peter-Monnik-Weg 4, 23562 Lübeck, Germany; al.hutfilz@uni-luebeck.de (A.H.); dirk.theisenkunde@uni-luebeck.com (D.T.-K.); ralf.brinkmann@uni-luebeck.de (R.B.)

4 Universitätsklinik für Augenheilkunde, Inselspital, Bern University Hospital, University of Bern, Freiburgstrasse 4, 3010 Bern, Switzerland; ebneter.andreas@gmail.com

check for updates

Citation: Burri, C.; Hutfilz, A.; Grimm, L.; Salzmann, S.; Arnold, P.; Považay, B.; Meier, C.; Ebneter, A.; Theisen-Kunde, D.; Brinkmann, R. Dynamic OCT Signal Loss for Determining RPE Radiant Exposure Damage Thresholds in Microsecond Laser Microsurgery. Appl. Sci. 2021, 11, 5535. https://doi.org/10.3390/ app11125535

Academic Editors:

Angelika Unterhuber and

Marco Andreana

Received: 14 April 2021

Accepted: 7 June 2021

Published: 15 June 2021

Publisher's Note: MDPI stays neutral with regard to jurisdictional claims in published maps and institutional affiliations.

Copyright: () 2021 by the authors. Licensee MDPI, Basel, Switzerland. This article is an open access article distributed under the terms and conditions of the Creative Commons Attribution (CC BY) license (https:// creativecommons.org/licenses/by/ $4.0 /)$.
5 Institute of Biomedical Optics, University of Lübeck, Peter-Monnik-Weg 4, 23562 Lübeck, Germany

* Correspondence: christian.burri@bfh.ch

Featured Application: Real-time dosimetry of selective retina therapy (SRT) using spectral-domain optical coherence tomography. SRT is a gentle laser treatment method for various diseases of the fundus (i.e., central serous chorioretinopathy and diabetic macular edema) associated with reduced retinal pigment epithelium function.

Abstract: Optical microsurgery of the retinal pigment epithelium (RPE) requires reliable real-time dosimetry to prevent unwanted overexposure of the neuroretina. The system used in this experiment implements optical coherence tomography (OCT) to detect the intentional elimination of RPE cells. We evaluated the performance of OCT dosimetry in terms of its ability to detect RPE cell damage caused by microsecond laser pulses of varying duration. Therefore, ex-vivo porcine RPE choroid sclera explants were embedded in an artificial eye and exposed to single laser pulses of 2-20 $\mu$ s duration (wavelength: $532 \mathrm{~nm}$, exposure area: $120 \times 120 \mu \mathrm{m}^{2}$, intensity modulation factor: 1.3). Simultaneously, time-resolved OCT M-scans were recorded (central wavelength: $870 \mathrm{~nm}$, scan rate: $33 \mathrm{kHz}$ ). Post-irradiation, RPE cell damage was quantified using a calcein-AM viability assay and compared with an OCT-dosimetry algorithm. The results of our experiments show that the OCTbased analysis successfully predicts RPE cell damage. At its optimal operating point, the algorithm achieved a sensitivity of $89 \%$ and specificity of $94 \%$ for pulses of $6 \mu$ s duration and demonstrated the ability to precisely control radiant exposure of a wide range of pulse durations towards selective real-time laser microsurgery.

Keywords: selective retina therapy; viability assay; photocoagulation; microbubble formation; thermomechanical damage; fringe washout; coherence-loss

\section{Introduction}

In the early 1950s, light coagulation was introduced as an outstanding optical therapeutic tool for remote tissue manipulation to prevent retinal detachment after retinal break formation [1]. The resulting technique is known today as laser photocoagulation (LPC) and is widely used as a treatment modality. In addition to its application for retinal detachment, LPC was found to be beneficial for ablative treatment of diabetic retinopathy [2], 
diabetic macular edema [3], central serous retinopathy [4] and other retinal pathologies. In LPC, millisecond laser-pulsed irradiation is absorbed by tissue pigments and converted to heat, causing photothermal denaturation. The delicate multi-layer retinal structure, consisting of the retinal pigment epithelium (RPE), Bruch's membrane, choriocapillaris and the otherwise healthy overlying neuroretina, including the sensitive photoreceptor cells, are collaterally damaged by laser pulses in the millisecond range, due to the relatively slow heating process and the relatively fast heat dissipation into the directly unheated neighboring tissue. This indirect heating resulting from excessive power in turn leads to scotoma, reduced night vision and disruption of the retinal anatomy through scarring $[5,6]$. Therefore, it is not possible to use LPC near the fovea because of the high risk of permanent loss of central vision $[7,8]$. These disadvantages have led to the development of new laser treatment modalities, which aim to produce specific therapeutic results without damaging photoreceptors and the neural retina by selectively acting on target structures. Among these treatment techniques, selective retina therapy (SRT), which targets only the prismatic retinal pigment epithelial cell monolayer, is particularly suitable [9]. The treatment is intended to selectively destroy a portion of defective RPE cells and utilize the regenerative abilities of the epithelium to recover its original structure. The targeted apoptotic RPE cells are cleared by phagocytosis, while extracellular debris is cleared by macrophages. Migration and proliferation of healthy neighboring RPE cells follow [10,11]. The stimulation of RPE cell migration is hypothesized to finally lead to improved metabolism at the target sites [9], which has been reported to have beneficial effects for patients suffering from RPE-related retinal diseases such as central serous chorioretinopathy [12]. The basis for this selective RPE damage is provided by the intracellular melanosomes, which despite the $<10 \mu \mathrm{m}$ thickness of the RPE monolayer [13], absorb about $50 \%$ of the incident light in the green spectral range. Within the thermal confinement of SRT, peak temperatures initiate microsecond-lifetime microbubble formation (MBF) on the melanosome's surface [14]. The rapid mechanical expansion and collapse of these microbubbles causes RPE cell-wall disruption, followed by immediate or delayed cell death [15].

Nevertheless, in practice, achieving selective RPE cell damage without damaging the surrounding tissue via heat diffusion is challenging due to the RPE's strongly varying melanin concentration. A successful SRT application is essentially based on the following two factors: first, the applied laser pulse duration, and second, accurate real-time energy dosing that depends on radiant exposure and local RPE absorptivity.

Regarding pulse durations, different experiments have shown that RPE cell death coincides with MBF for laser pulse durations up to $50 \mu \mathrm{s}[16,17]$. Lee et al. were able to show that, in the case of 1,5 and $10 \mu$ s laser pulse duration, more than $95 \%$ of dead cells were associated with MBF, whereas the ratio decreased to $65 \%$ and $45 \%$ for longer 20 and $40 \mu$ s pulses, respectively [18]. This suggests that pulse durations longer than $20 \mu \mathrm{s}$ delineate the limit of thermal confinement for SRT; beyond this, the technique appears impractical even with the use of real-time dosimetry. By contrast, SRT laser pulses in the microsecond time regime $(1.7 \mu \mathrm{s}, 5 \mu \mathrm{s})$ appear favorable compared to nanosecond pulses $(8 \mathrm{~ns}, 200 \mathrm{~ns})$ in preventing unintentional retinal damage $[19,20]$. Recent clinical studies have shown promising results using SRT with a series of 15 to 30 pulses, at $1.7 \mu$ s pulse duration and repetition rates of $100 \mathrm{~Hz}[12,21,22]$.

For clinical SRT application, energy dosing (i.e., absorption-corrected radiant exposure) is essential. In order to conserve photoreceptor integrity, SRT irradiation must be kept within a small treatment window close above the MBF threshold, so as to avoid insufficient or excessive exposure that would result in negligible or negative effects. This threshold varies both between patients and within retinal regions of individual patients, because of the optical transmission differences and light absorption variability at the fundus due to the localized melanosome density [5]. Furthermore, by definition, the treatment window of SRT limits induced tissue damage to the RPE within, and thus is undetectable during visual inspection of the fundus. Currently, selective RPE damage caused by microsecond laser irradiation is only detectable with biochemical methods such as fundus fluorescein angiog- 
raphy (FFA). This has two major drawbacks: first, it is invasive-due to the intravenously injected dye-which introduces a risk of anaphylactic shock, and second, it has a significant delay, since FFA can only be performed post-SRT-treatment (and only once per day). Hence, several approaches for real-time MBF detection and real-time laser exposure control for individual lesions (feedback-controlled irradiation) are under development. Methods such as measuring the increased reflectance at the bubble surface via backscattered light or capturing the ultrasonic emission of vaporization to detect the appearance of MBF have been successfully tested $[17,23]$.

An alternative dosimetry approach is to use spectral-domain optical coherence tomography (SD-OCT) simultaneously with SRT to observe MBF formation. This method was described first by Steiner et al., who indirectly detected tissue effects of laser pulses as signal changes in time-resolved SD-OCT A-scans (M-scans), which correspond to a change in the local reflectivity of tissue [24-26]. The detailed origin of this signal loss is still debated, but the currently favored hypothesis explains it as "coherent fringe washouts" resulting from the axial motion of the retina due to MBF. This coherence decorrelation is assumed to result from rapid fluctuations of the spectral phase, which are faster than the integration time of the detector and therefore larger than $2 \pi$ within the acquisition time of the interferometric measurement (typically 10 to $30 \mu \mathrm{s})$. Successively, the timeaveraged heterodyne signal-the spectral fringe pattern-diminishes, while the homodyne components-the spectral shape-are almost unaffected. This behavior indicates that neither the local nor the overall reflectivity is altered, and sensor overexposure can be ruled out as an origin of the phenomenon. Stronger changes, caused by fast variations in reflectance, i.e., as expected from larger quickly expanding or collapsing microbubbles with their high refractive index jumps between gas and fluid, also result in a loss of the homodyne signal. However, MBF also affects the homodyne spectral shape at the MBF-location, while the resulting sonic vibrations spread out laterally and axially for multiple tens of micrometers, thereby forming an enlarged volume of rapid oscillations where OCT signals get cloaked. The resulting significant sensitivity advantage of the OCT-approach compared to direct reflectance sensing potentially enables irradiation-effect estimation even at levels beneath the cell-lethality threshold.

Based on this model, using signal changes of SD-OCT M-scans, Kaufmann et al. were able to precisely predict RPE lesions post-treatment in ex vivo porcine eyes $[27,28]$. Furthermore, Kaufmann et al. showed that SRT in ramp mode, combined with fast algorithm-based SD-OCT treatment termination, could provide reliable SRT dosing and prevent overtreatment. Recently, Lee et al. were able to show the same effect of abrupt signal changes with a slightly different OCT acquisition scheme, which utilized a wavelength scanning radiation source termed "swept-source" (SS-OCT) [29]. These authors also aimed to extract local temperature information from speckle variations, to support the hypothesis that this occurred due to strictly localized heating of melanosomes without major temperature increases in neighboring regions [29].

With this tool for monitoring stress at the cellular scale, the optimal pulse parameters leading to the desired cellular damage can be explored in more detail. To enable automated, highly reliable, selective RPE-level laser treatment, with maximum clinical control, it is necessary to further analyze how well the OCT signal can predict different biological outcomes. The aim of the current work was therefore to investigate the range of pulse durations (from 2 to $20 \mu \mathrm{s}$ ) in more detail with respect to the threshold radiant exposure for RPE cell damage. A fluorescence-based vitality stain (calcein-AM-assay, median effective dose for RPE cell damage: $E D_{50}^{R P E}$ ) was used directly after laser irradiation. The fluorescence change index of cell damage was compared with fringe washouts observed in SD-OCT M-scans (median effective dose for fringe washouts: $E D_{50}^{O C T}$ ). If the threshold for fringe washouts equals the threshold for RPE cell damage, then the damage can be assumed to be primarily thermomechanical, and thus likely to result in the desired selective RPE damage. Such knowledge may allow the pulse duration regime for SRT to shift to higher pulse durations. 
To test the hypothesis that SD-OCT has the potential to be used for real-time dosimetry of RPE cell damage, a specially designed system [30] was employed to investigate OCT $\mathrm{M}$-scan fringe washouts during microsecond laser irradiation. Successively, the therapeutic window required to achieve a selective effect in the RPE was examined to investigate if it could be expanded from $1.7 \mu \mathrm{s}$ - as previously used in Q-switched laser systems (i.e., R:GEN, Lutronic, Gyeonggi-do, Korea) - to longer pulse durations, which would allow the use of smaller, better controllable and more flexible SRT laser sources.

\section{Materials and Methods}

\subsection{Treatment and Monitoring System}

The so-called Spectralis Centaurus system (HuCE-optoLab, Bern University of Applied Sciences, Biel, $\mathrm{CH}$ ) was used for the experiments. It consists of an opto-mechanically upgraded commercial diagnostic imaging platform (SPECTRALIS HRA+OCT, Heidelberg Engineering, Heidelberg, DE) and is extended with an experimental SRT laser (modified MERILAS 532 shortpulse, Meridian, Thun, CH). The system utilizes SD-OCT as widely used for diagnosis of retinal diseases by capturing cross-sectional and volumetric images (B- and C-scans). It features the ability of intervention planning via a coaxially integrated confocal scanning laser ophthalmoscope (cSLO). Furthermore, it can be operated in the so-called M-scan mode (motion mode) to measure time-resolved sequences of A-scans at the point of the therapy laser application, thereby revealing depth-resolved temporal signal fluctuations, e.g., tissue movements during laser exposure (Figure 1).

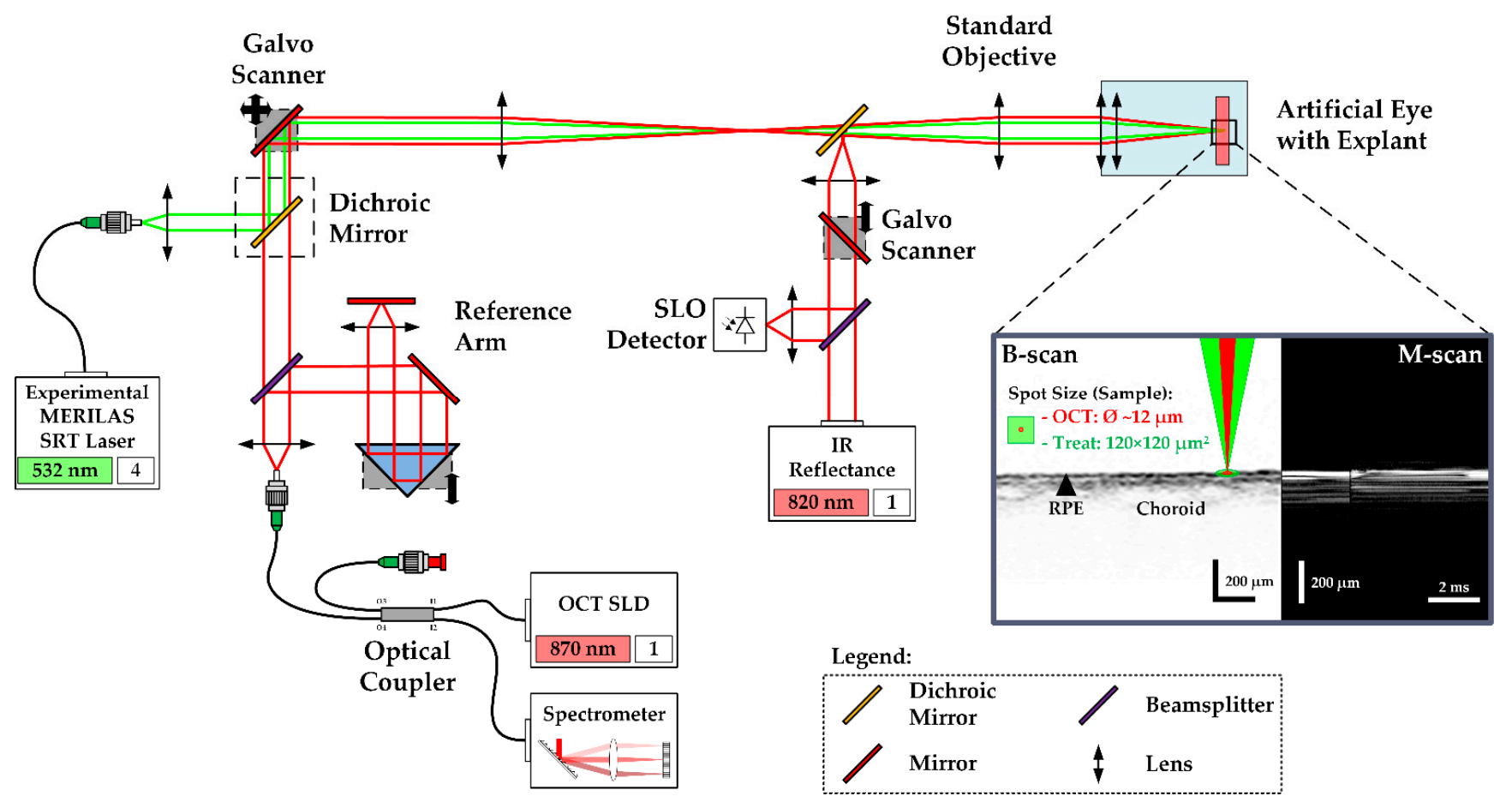

Figure 1. Setup and functionality of the Spectralis Centaurus system: Treatment laser (532 nm) and SD-OCT (870 nm) path are combined by a dichroic mirror (black-dashed rectangle). Retina scanning is performed by a galvanometric scanner. A chromatically corrected objective focuses the collinear treatment and OCT laser beams. The reflected light is separately guided towards the spectrometer and a fast diode. The fast scanning IR cSLO $(820 \mathrm{~nm})$ eases treatment planning by displaying a fundus image + where the treatment pattern can be placed interactively. The porcine RPE-choroid-sclera explants were mounted in an upright artificial eye (optical power: $65 \mathrm{dpt}$ ).

The experimental SRT laser utilizes optically pumped semiconductor laser (OPSL) technology and emits light at $532 \mathrm{~nm}$ wavelength, with a peak power of $30 \mathrm{~W}$. It can be operated in two different modes: CW mode and SRT mode. In CW mode, the laser operates like a conventional LPC laser, which was used to create marker lesions on the sample. 
In SRT mode, the laser emits single pulses or bursts of laser pulses of 2 to $20 \mu$ s pulse duration at a repetition rate of $100 \mathrm{~Hz}$. The laser radiation is transmitted via a square core multimode fiber into the camera head of the system, to laterally homogenize the beam profile, where it is combined with the OCT laser beam by a dichroic mirror as depicted in Figure 1. The projected top-hat square beam profile measured $120 \times 120 \mu^{2}$ on the RPE-choroid-sclera explant's surface. Prior to the tissue experiments, the top-hat square beam profile was rated regarding its spatial intensity modulation factor (IMF). The IMF describes the ratio of maximum to mean radiant exposure over the beam profile and was first introduced by Framme et al. in 2002 as a speckle factor [31]. IMF $=1$ corresponds to perfectly homogenous, top-hat radiant exposure. To calculate the IMF, the treatment laser beam was imaged with a magnification of 9.81 onto a beam analyzer camera (BC 106 VIS, Thorlabs Inc., Newton, NJ, USA)) behind the laser aperture of the system. An IMF = 1.3 was determined, which indicates a high-quality top-hat beam profile [32].

The diagnostic imaging part of the system is based on spectral-domain (SD), also called Fourier-domain (FD) OCT, which allows high-speed and high-resolution imaging of the retina with an interferometric high-speed spectrometer, enabling simultaneous acquisition of a depth or amplitude mode (A) scan. The super-luminescence diode of the SD-OCT laser emits infrared radiation centered at $870 \mathrm{~nm}$ wavelength and $73 \mathrm{~nm}(840-913 \mathrm{~nm})$ spectral bandwidth. The OCT system is operated at a rate of $33 \mathrm{kHz}$ and $30.3 \mu$ s per A-scan integration time. In B-scan mode, the beam scans across the retina, producing a cross-sectional image. For volumetric, three-dimensional retinal imaging (C-scan), up to 768 orthogonally equally spaced B-scans are acquired sequentially.

In the experiment, treatment laser exposures for the creation of RPE lesions were correlated to signal variations in SD-OCT M-scans, consisting of 256 sequentially recorded A-scans (at $7.76 \mathrm{~ms}$ total acquisition time per M-Scan). For each lesion, seven consecutive M-scans were recorded, which leads to time-resolved records of 37.24 ms duration. The treatment laser was triggered to emit at the first third of the M-scan time period. The OCT beam waist with a diameter of $12 \mu \mathrm{m}$ was adjusted to the center of the treatment spot.

\subsection{Treatment Pattern and Irradiation}

As depicted in Figure 2a, the outer rim of the laser test pattern contained 25 LPC marker lesions. These marker lesions were applied in CW mode $(200 \mathrm{~ms}$ pulse duration and $200 \mathrm{~mW}$ pulse power). The marker lesions served as an orientation guide in the evaluation process of the RPE explant. The microsecond laser probe region was placed inside the demarcation frame consisting of a pattern of $10 \times 10$ lesions. Laser pulses with durations of $2,6,10,14$ and $20 \mu \mathrm{s}$ with increasing energy were applied. The exact radiant exposure values for each lesion can be found in Table A1 in Appendix A. The pulse energy was measured with a calibrated energy meter (J-10MB-LE, Coherent, Santa Clara, CA, USA) prior to the experiments in front of the laser aperture of the system. The energy meter has a measurement accuracy of $2.8 \%$ at $532 \mathrm{~nm}$. The radiant exposures reported in this work were calculated using the following equation (using a spot size on the explant of $\mathrm{A}=120 \times 120 \mu \mathrm{m}^{2}$ (Figure $2 \mathrm{~b}$ ) and an IMF of 1.3):

$$
H=\frac{E_{\text {pulse }}}{A} \cdot I M F
$$

Figure 2a shows that the pulse duration was increased from top to bottom ( 2 to $20 \mu \mathrm{s}$ ), and the radiant exposure was increased from left $\left(135 \mathrm{~mJ} / \mathrm{cm}^{2} \hat{=} 15 \mu \mathrm{J}\right)$ to right (maximum: $1354 \mathrm{~mJ} / \mathrm{cm}^{2} \hat{=} 150 \mu \mathrm{J}$ at $20 \mu \mathrm{s}$ ). Every laser setting appeared twice within the pattern to investigate reproducibility due to local variability in the RPE melanin distribution. The useful range of target energies for each pulse duration within the treatment pattern was pre-evaluated by multiple RPE explant tests. 

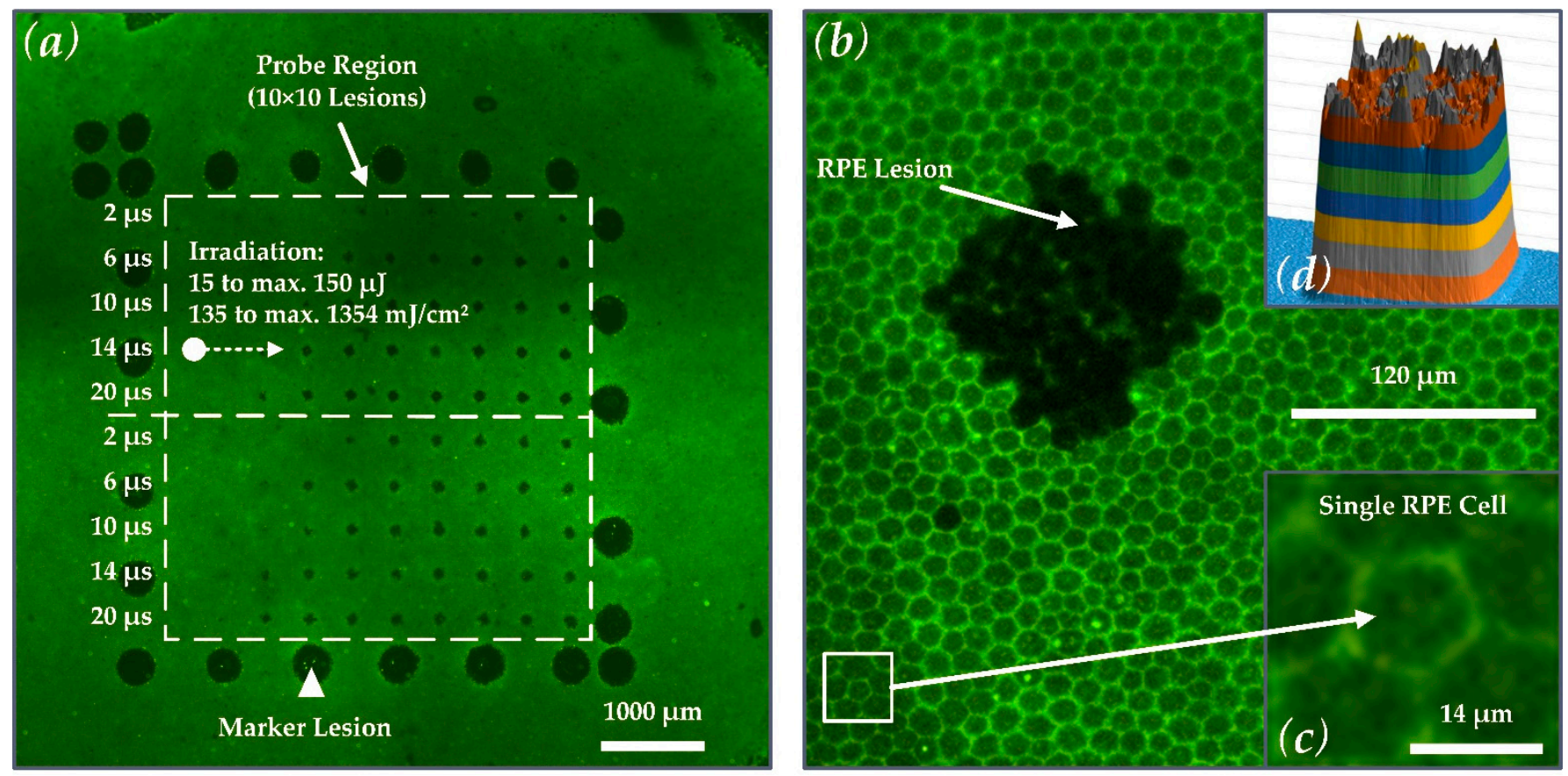

Figure 2. (a) Calcein-AM image of porcine RPE-choroid-sclera explant No. 2 after laser irradiation, showing the large LPC marker lesions (white triangle) and lesions in the probe region (white-dashed rectangle; $10 \times 10$ lesions) where the microsecond treatment pattern was applied. The marker lesions served as orientation guide in the evaluation process of the experiments. (b) Close-up view of the explant showing a rectangular lesion in the RPE applied with microsecond laser irradiation with a spot of size $120 \times 120 \mathrm{\mu m}^{2}$. (c) When viewed en face, the RPE single-cellular layered hexagonal shape is revealed. Typically, the single porcine RPE cells measure about $14 \mu \mathrm{m}$ in diameter, which corresponds to the size of RPE cells in the human fovea [33]. (d) The strongly pronounced rectangular lesion is directly related to the high-quality square top-hat laser spot with an IMF of 1.3.

\subsection{Explant Preparation and Artificial Eye}

In total, 13 RPE-choroid-sclera explants from enucleated porcine eyes were processed. The samples were stripped from anterior parts of the eye, lens and vitreous body and maintained in a Dulbecco's Modified Eagle Medium (DMEM, high glucose, Merck KgaA, Darmstadt, Germany) mixed with $10 \%$ porcine serum, $1 \mathrm{mM}$ sodium pyruvate and antibiotic antimicrobial agents on a heating plate at $37^{\circ} \mathrm{C}$ during the test day. This method of tissue preparation has been previously described by Miura et al. [34].

For irradiation, the RPE was retained in a customized artificial eye (Medical Laser Center Lübeck, Lübeck, Germany), kept at room temperature and placed in front of the laser system. It consisted of a cuvette for phosphate-buffered saline (PBS) solution, an explant holder, an integrated hard lens as cornea at the front (BC $7.8 \mathrm{mM}$, PAUL, Wöhlk, Schönkirchen, Germany) and a double-convex lens (32016, Edmund Optics Ltd., York, UK) as crystalline lens replacement with $65 \mathrm{dpt}$ of optical power.

\subsection{Calcein-AM Assay and Binary Evaluation of the RPE Damage}

The viability of the RPE cells was tested by a calcein-AM assay directly after microsecond laser irradiation. Calcein-AM diffuses into the cell and is converted by intracellular esterases into calcein that fluorescents under blue light. Dead cells exhibit no esterase activity and therefore show no fluorescence. The RPE-choroid-sclera explants were incubated with $3 \mathrm{mM}$ calcein-AM in PBS for $15 \mathrm{~min}$. at room temperature. The viable-lethal analysis was conducted using a fluorescence microscope (Eclipse Ti-E, Nikon, Tokio, Japan) and a FITC-filter (excitation wavelength: $465-495 \mathrm{~nm}$, dichroic for $505 \mathrm{~nm}$, barrier filter at $515-555 \mathrm{~nm}$ ). The cellular calcein-fluorescence assay had a binary outcome. In the case of visible SRT-induced damage, lesions were evaluated with 1 and in the case of no damage 
with 0 . Visible damage was defined as a cluster of at least three non-fluorescing RPE cells in the exposure region, which appear dark in fluorescence microscopy. RPE damage exposure thresholds were determined via Probit-Plot analysis. The calculation was performed with Origin 2018 (OriginLab Corporation, Northampton, MA, USA) utilizing the Levenberg Marquardt iteration algorithm to fit with a $\chi^{2}$-tolerance-value of $1 \times 10^{-9}$ within up to 400 iteration steps. The Probit-fit provides the irradiation threshold (ED 50 -value). An $E_{50}$ irradiation value means that $50 \%$ of treatments at this irradiation level depict RPE lesions. $\mathrm{ED}_{15}$ and $\mathrm{ED}_{85}$ values were calculated to visualize the width of the fitted normal distribution in a logarithmic covariant basis [35]. Post-processing of the fluorescence microscopies was accomplished with the Fiji image processing package distribution of ImageJ [36].

\subsection{Binary Evaluation of M-Scan Fringe Washouts}

The SD-OCT M-Scans were examined for fringe washout using an automated OCT dosimetry algorithm. In the evaluation, which took place post-treatment, the automated OCT dosimetry algorithm detects fringe washouts in three basic steps, whereas each Mscan is processed separately (Figure 3). First, the algorithm performs an intensity scaling and gamma correction for contrast enhancement and sums up the intensity values of each A-scan within the M-scan. Second, the data are convolved with a dedicated filter kernel to create a peak response to possible fringe washouts. Third, a dynamic threshold that adapts to noise and intensity differences is applied to the processed M-scans to identify fringe washouts based on the peak response from step two. According to the equation depicted in step No. 3. in Figure 3, the threshold $(T)$ is based on a central tendency measure $(M)$ of the processed M-scan signal, a dispersion measure $(D)$ of the central tendency and a sensitivity factor $(\kappa)$ referred to as the $\kappa$-value. The sensitivity factor describes that the value of the peak (fringe washout) must be $\kappa$ times the dispersion D away from the central tendency $\mathrm{M}[37]$.

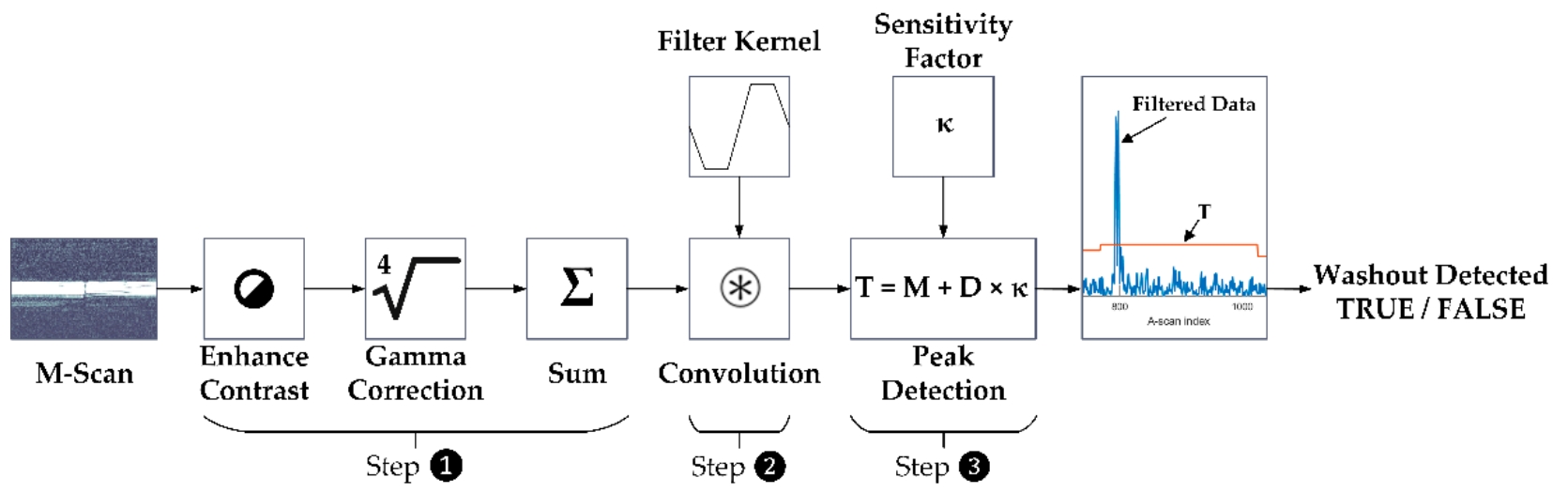

Figure 3. Flowchart describing how an M-scan is processed by the automated OCT dosimetry algorithm. Step 1: intensity scaling, gamma correction and intensity summation. Step 2: signal convolution with filter kernel. Step 3: dynamic threshold for peak detection (fringe washout).

In view of a future in vivo application, the $\kappa$-value was defined as a variable parameter that statically modulates the response and that must be selected manually by the operator. This will give the dosimetry algorithm a certain flexibility to adapt to different anatomical and optical eye conditions of patients. To check which algorithm setting could be suitable for future in vivo applications, the M-scan data were analyzed for fringe washouts with different $\kappa$-values. Therefore, all acquired $M$-scans were evaluated with $\kappa$-values ranging from 6 to $40(\kappa=6,9,10,11,12,13,14,15,20$ and 40$)$. The selection of this range is based on previous Matthews correlation coefficient classifications [37]. The outcome of the M-scan evaluation was compared to the binary calcein-AM evaluation of the porcine RPE-choroid-sclera explants and classified by using the confusion matrix presented in the following section. Statistical measures were then analyzed via a receiver operating 
characteristic (ROC) curve. The ROC of an algorithm shows its performance as a tradeoff between selectivity and sensitivity, with the optimum performance in the top-left corner of the graph. Therefore, we used the ROC curve to determine the dosimetry algorithms optimal $\kappa$-value by checking the optimal operating point (OOP) for each pulse duration. Furthermore, we calculated the area under the ROC curve (AUC) in order to determine for which pulse duration, the dosimetry algorithm showed the best overall performance.

\subsection{Statistical Evaluation of OCT M-Scan and Calcein AM Data}

The ground truth for the statistical evaluation is based on the binary calcein-AM evaluation of the porcine RPE-choroid-sclera explants and the hypothesis that RPE lesions due to MBF lead to fringe washouts in SD-OCT M-scans.

For statistical evaluation, the occurrence of fringe washouts (Predictive Class) is compared to the damage outcome on the explants (Actual Class) by using a confusion matrix. The confusion matrix shown in Figure 4 features four cardinalities: true positive (TP), true negative (TN), false positive (FP) and false negative (FN). Positive and negative refer to the prediction made by SD-OCT M-scans of whether an RPE lesion was created, based on the presence of fringe washouts, while true and false evaluate the correctness of this statement.

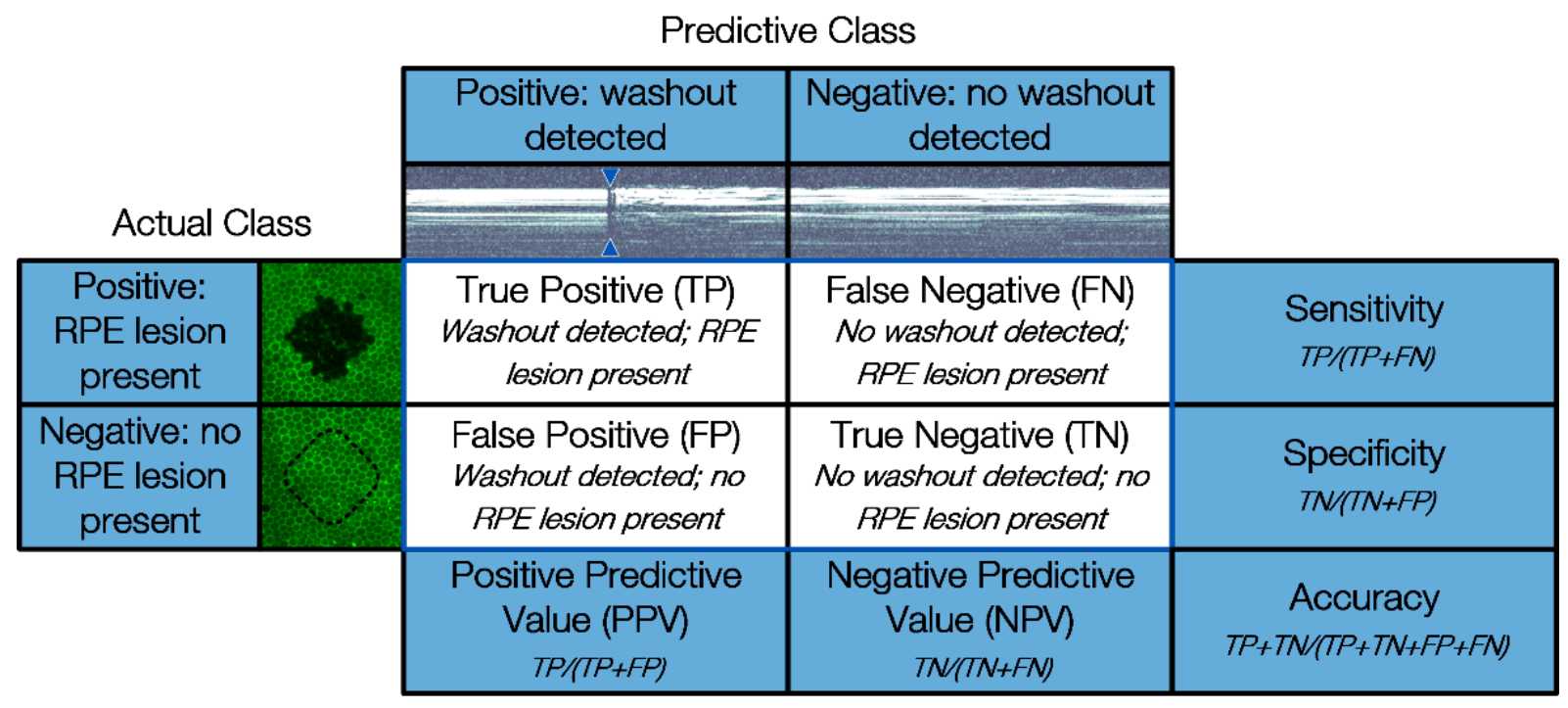

Figure 4. Confusion matrix used to compare fringe washouts in SD-OCT M-scans (Predictive Class) with the treatment outcome of the viability tests with calcein-AM and the binary evaluation of the porcine RPE-choroid-sclera explants (Actual Class).

Based on the four cardinalities of the confusion matrix, several statistical measures can be derived to present the overall device performance in a straightforward fashion. In our case, the sensitivity, specificity, accuracy, positive predictive value (PPV) and negative predictive value (NPV) were calculated.

\section{Results}

Figure 5 presents the calcein-AM and SD-OCT M-scan evaluation based on Explant No. 6 as an example. The experimental results of the Probit-fit analysis for the different applied laser pulse durations are reported, together with the SD-OCT M-Scan evaluation. The quantity of laser lesions applied is demonstrated to be sufficient for Probit-fitting. Out of 13 RPE-choroid-sclera explants that received a total of 1300 SRT laser spots, 10 explants and 1000 treatment spots were included in the evaluation (three explants were excluded due to damage to the RPE during the preparation for staining). Finally, an overall statistical evaluation is presented, with 200 measurements per pulse duration. 

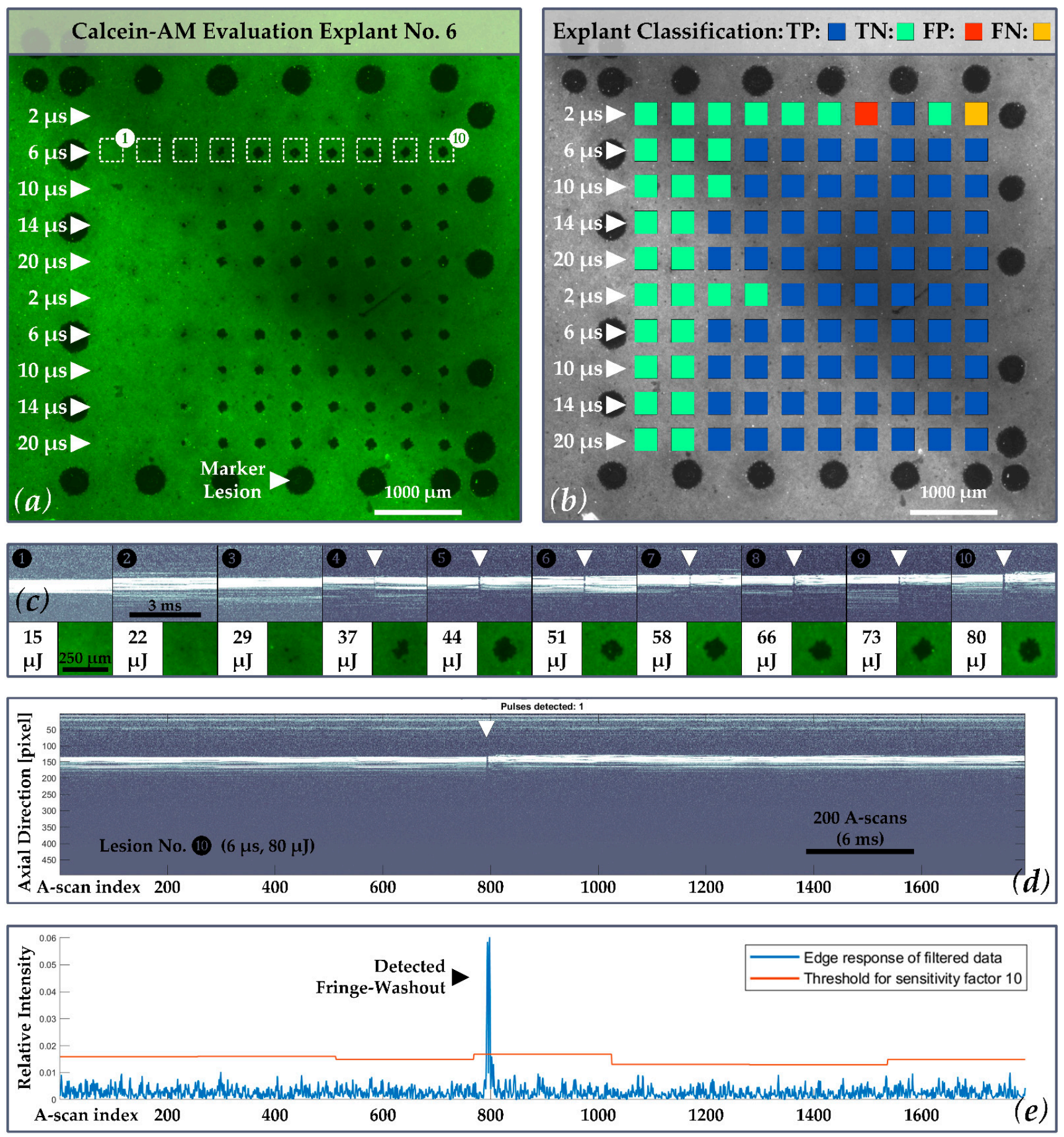

Figure 5. (a) Calcein-AM image of porcine RPE-choroid-sclera explant No. 6. The second treatment line within the probe region is emphasized (white-dashed rectangles 1-10), in which laser pulses of $6 \mu$ s pulse duration were applied; (b) exemplary explant classification according to the confusion matrix with 69 True Positive (TP = Blue), 29 True Negative TN = Cyan), 1 False Positive (FP = Red), 1 False Negative (FN = Yellow) for each individual spot evaluated by the algorithm ( $\kappa$-value of 10 ). In this case, a sensitivity of $99 \%$, a specificity of $97 \%$ and an accuracy of $98 \%$ was achieved; (c) Line two in, which laser pulses of $6 \mu$ s pulse duration were applied, is shown in more detail and with the corresponding SD-OCT M-scans for each lesion: no fringe washout and no RPE lesion is present for lesions No. 1 to lesion No. $3 .\left(80 \mathrm{~mJ} / \mathrm{cm}^{2} \hat{=} 15 \mu \mathrm{J}\right.$, $\left.118 \mathrm{~mJ} / \mathrm{cm}^{2} \hat{=} 22 \mu \mathrm{J}, 155 \mathrm{~mJ} / \mathrm{cm}^{2} \hat{=} 29 \mu \mathrm{J}\right)$. Washouts and RPE lesions start to occur in this sample for a radiant exposure of $198 \mathrm{~mJ} / \mathrm{cm}^{2} \hat{=} 37 \mu \mathrm{J}$ at $6 \mu$ s pulse duration; (d) Complete SD-OCT M-scan record for lesion No. $10\left(427 \mathrm{~mJ} / \mathrm{cm}^{2} \hat{=} 80 \mu \mathrm{J}\right)$ showing a fringe washout around A-scan number 800 . For each lesion, an M-scan of 54 ms duration was recorded, containing 1792 A-scans (SD-OCT scan rate $=33 \mathrm{kHz}$ ); (e) Corresponding evaluation by the algorithm with detected washout. 


\subsection{Exemplary Explant Evaluation}

Figure 5 shows an exemplar calcein-AM and SD-OCT M-scan evaluation of explant No. 6. As depicted in Figure 5a, the explant shows 25 LPC marker lesions as well as RPE lesions within the $10 \times 10$ probe region, whereby a specific damage threshold is visible. In the corresponding example presented in Figure $5 b$, a $\kappa$-value of 10 was used by the dosimetry algorithm in order to represent all cases according to the confusion matrix. In this case, a sensitivity of $99 \%$, a specificity of $97 \%$ and an accuracy of $98 \%$ were reached (TP: 69, TN: 29, FP: 1, FN: 1; PPV: 99\%, NPV: 97\%).

\subsection{RPE Cell Damage and M-Scan Signal Washout Thresholds for Single Pulse Irradiation}

Overall, the binary evaluation resulted in 632 visible RPE lesions. The resulting $E D_{50}$ values (radiant exposure thresholds for RPE cell damage $\left(E D_{50}^{R P E}\right)$ and M-scan fringe washouts $\left(E D_{50}^{O C T}\right)$ by single irradiation for different pulse duration) from the viability tests are summarized in Figure 6. The exact threshold values per laser pulse duration can be found in Table A2 in Appendix A.

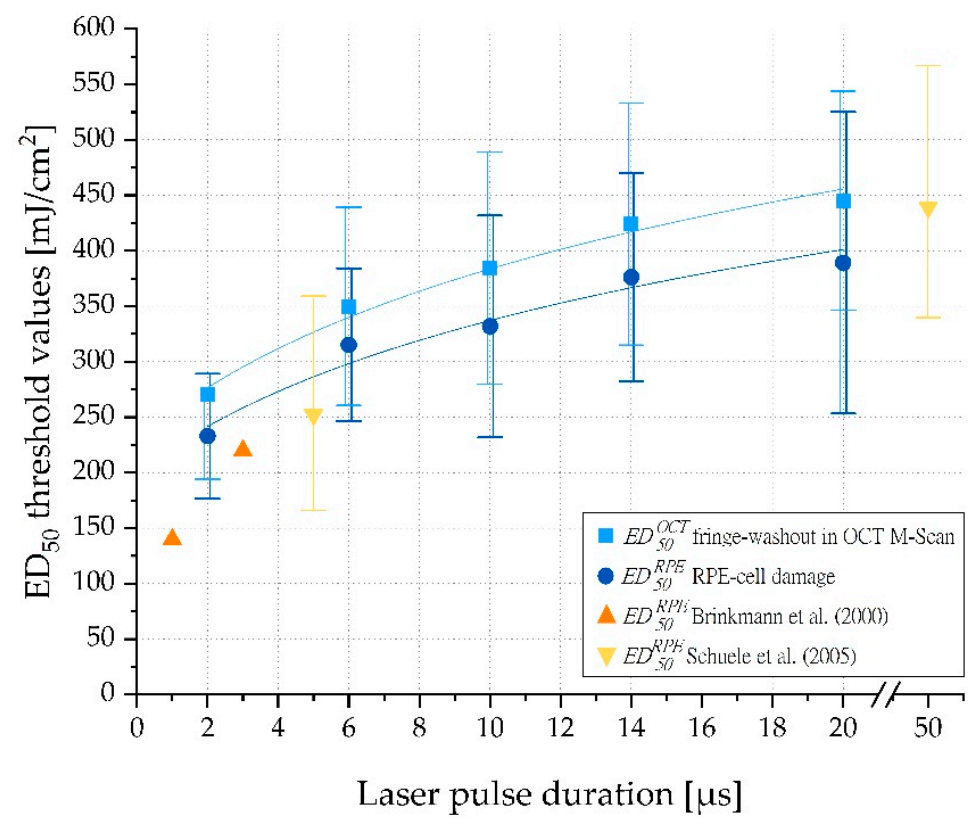

Figure 6. $E D_{50}$ threshold exposures $\left(\mathrm{mJ} / \mathrm{cm}^{2}\right)$ for RPE cell damage $\left(E D_{50}^{R P E}\right)$ and fringe washouts in OCT M-scans $\left(E D_{50}^{O C T}\right)$ for single-pulse laser irradiation $(2 \mu \mathrm{s}, 6 \mu \mathrm{s}, 10 \mu \mathrm{s}, 14 \mu \mathrm{s}$ and $20 \mu \mathrm{s})$. The error bars indicate the width of the normal distribution $\left(\mathrm{ED}_{15}\right.$ and $\left.\mathrm{ED}_{85}\right)$. The chart is supplemented with values from Brinkmann et al. [38] and Schuele et al. [39].

\subsection{Optimal Operating Point OCT Dosimetry Algorithm}

Fringe washouts in SD-OCT M-scans were evaluated with the automated dosimetry algorithm for different $\kappa$-values ranging from 6 to 40 and compared to levels of RPE cell damage. Data comparison took place by using the confusion matrix presented in Figure 4. The resulting statistical measures (sensitivity and specificity) are described by the ROC curve in Figure 7. The higher the AUC, the better the algorithm predicts RPE damage based on fringe washouts. The best performance was obtained for a pulse duration of $6 \mu \mathrm{s}(\mathrm{AUC}=0.97)$ and $14 \mu \mathrm{s}(\mathrm{AUC}=0.97)$. Very similar results were obtained for pulse durations of $20 \mu \mathrm{s}(\mathrm{AUC}=0.96)$ and $10 \mu \mathrm{s}(\mathrm{AUC}=0.93)$. Notably, for a pulse duration of $2 \mu \mathrm{s}$, the performance was lower $(\mathrm{AUC}=0.85)$. 

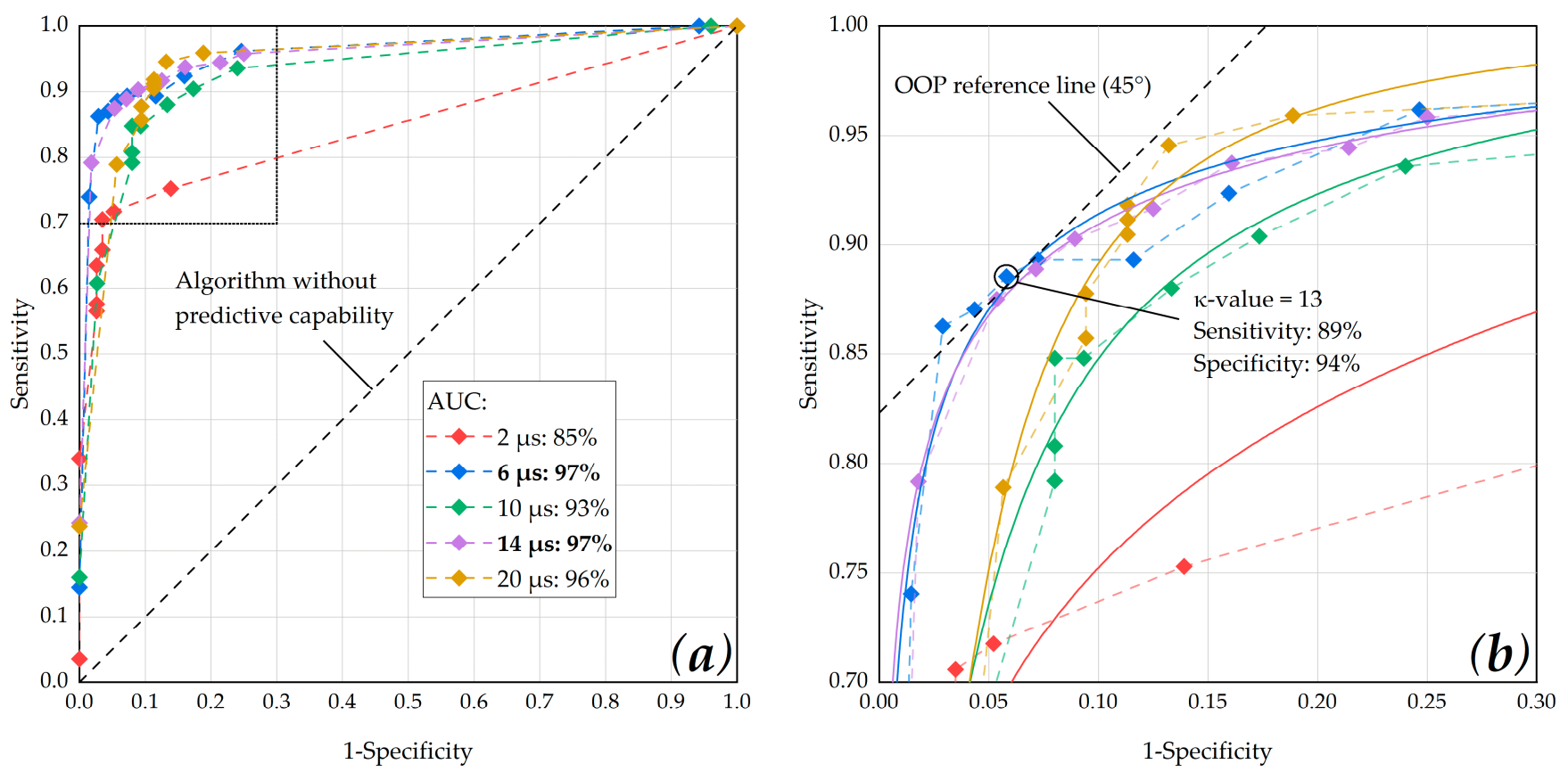

Figure 7. (a) ROC curve of the dosimetry algorithm showing the performance at different pulse durations with respect to different $\kappa$-values. The best performance was obtained for a pulse duration of $6 \mu$ s (AUC $=0.97$ ) and $14 \mu \mathrm{s}$ (AUC 0.97); (b) in order to define the optimal operating point (OOP), the data points were fitted using OriginLab's Logistic function (Logistic-FitFunc, indicated as solid lines). The data point that is closest to the $45^{\circ} \mathrm{OOP}$ reference line was determined as $\kappa$-value of 13 at a laser pulse duration of $6 \mu$ s (sensitivity: 0.89 , specificity: 0.94 ).

The OOP of the dosimetry algorithm was interpreted by analyzing the upper left section of the ROC curve (Figure $7 \mathrm{~b}$ ) and by fitting the data points using OriginLab's Logistic function (Logistic-FitFunc). The data point that was closest to the $45^{\circ} \mathrm{OOP}$ reference line was determined as $\kappa$-value $=13$ at a laser pulse duration of $6 \mu \mathrm{s}$ (sensitivity: 0.89 , specificity: 0.94 ) according to the assumption that specificity and sensitivity are weighted equally. Highly similar results were obtained for $\kappa$-values of 14 (sensitivity: 0.87, specificity: 0.96) and 12 (sensitivity: 0.89 , specificity: 0.93 ).

\subsection{Overall Statistical Evaluation}

Based on the previous OOP evaluation, an overall statistical evaluation for all pulse durations (at a $\kappa$-value of 13) is presented in Table 1 For this data set, the algorithm-based RPE cell damage evaluation achieved an overall sensitivity of $85 \%$ and an overall specificity of $93 \%$. The optimum sensitivity was achieved for laser pulses of 14 and $20 \mu$ s duration (sensitivity for both: $90 \%$ ). The optimum specificity was achieved for a laser pulse of $2 \mu \mathrm{s}$ duration (specificity: 97\%).

Table 1. Overall statistical evaluation for all pulse durations (OCT algorithm: $\kappa$-value of 13).

\begin{tabular}{c|ccccc|c}
\hline Pulse Duration & $\mathbf{2} \boldsymbol{\mu s}$ & $\mathbf{6} \boldsymbol{\mu s}$ & $\mathbf{1 0} \boldsymbol{\mu} \mathbf{s}$ & $\mathbf{1 4} \boldsymbol{\mu s}$ & $\mathbf{2 0} \boldsymbol{\mu s}$ & Overall \\
\hline TP & 54 & 116 & 106 & 130 & 133 & 539 \\
TN & 112 & 65 & 69 & 51 & 47 & 344 \\
FP & 3 & 4 & 6 & 5 & 6 & 24 \\
FN & 31 & 15 & 19 & 14 & 14 & 93 \\
\hline Sensitivity & 0.64 & 0.89 & 0.85 & 0.90 & 0.90 & 0.85 \\
Specificity & 0.97 & 0.94 & 0.92 & 0.91 & 0.89 & 0.93 \\
Accuracy & 0.83 & 0.91 & 0.88 & 0.91 & 0.90 & 0.88 \\
PPV & 0.95 & 0.97 & 0.95 & 0.96 & 0.96 & 0.96 \\
NPV & 0.78 & 0.81 & 0.78 & 0.78 & 0.77 & 0.79 \\
\hline
\end{tabular}




\section{Discussion}

We tested the hypothesis that SD-OCT has the potential to predict RPE cell damage after microsecond laser exposure. The Spectralis Centaurus system and an experimental SRT laser [30] were used to investigate OCT M-scan fringe washouts during microsecond laser irradiation by using laser pulses in the range of 2 to $20 \mu \mathrm{s}$.

As reported by Brinkmann et al. [38] and Schuele et al. [39], we confirmed that RPE radiant exposure damage thresholds increase with increasing pulse duration (Figure 6). Furthermore, our results for porcine RPE damage exposure thresholds were in good agreement with data of 1 and $3 \mu$ s laser pulses reported by Brinkmann et al. and 5 and $50 \mu$ s laser pulses found by Schuele et al. Recently published mean RPE damage exposure thresholds on lightly pigmented porcine eyes reported by Seifert et al. for laser pulses of 2 to $50 \mu$ s duration are also consistent with our findings for $2 \mu$ s pulses. However, for longer pulses, up to $20 \mu$ s pulse duration, the damage exposure thresholds reported by Seifert et al. were significantly higher than those we observed. This is consistent with the theory that for short pulses, particle absorption by single melanosomes dominates, while for longer pulses, the lower bulk absorption by melanosomes plays a major role [40]. In principle, absolute values should always be considered as rough guide values only, as they can vary greatly and the whole concept of a laser system that compensates for differences in melanin concentration across individuals or across species variations argues against the use of an optimum fixed absolute value.

Regarding evidence for the selectivity of longer pulse durations up to $20 \mu \mathrm{s}$, the results of Schuele et al. are particularly relevant. These authors showed that MBF is the dominant origin of RPE cell damage for pulses up to $50 \mu$ duration [39]. Lee et al. reached a similar conclusion [18]. Consequently, the transition for MBF-based to pure thermal RPE cell damage can thus be assumed to be in the range of 20 to $50 \mu$ s. Our results substantiate that thresholds for both RPE cell damage and OCT fringe washouts are approximately the same (Figures 6 and A1b). Therefore, it can be inferred that RPE cell damage up to a pulse duration of $20 \mu$ s is predominantly caused by MBF, and thus reliable dosimetry can be performed by monitoring localized MBF to enable SRT. In addition, OCT also detects vibrations in the proximity of the MBF and is sensitive enough to detect subliminal changes.

The results of the calcein-AM assay underline the necessity of dosimetry for ophthalmoscopically sub-visible laser therapies, such as SRT. In accordance with Schuele et al., we observed a large difference between the $\mathrm{ED}_{15}$ and $\mathrm{ED}_{85}$ values (e.g., $10 \mu \mathrm{s}: E D_{15}^{R P E}=25 \mu \mathrm{J}$, $E D_{85}^{R P E}=62 \mu \mathrm{J}, \Delta Q=37 \mu \mathrm{J} 334 \mathrm{~mJ} / \mathrm{cm}^{2}$ ), which is due in part to the high inter- and intra-individual melanin density variations in the different RPE samples [39]. Regarding the evaluation of our porcine RPE-choroid-sclera explants, practically all samples showed local radiant exposure damage threshold variability (Figure 8 ). This variation may be related to local differences in the melanin distribution of the RPE. While in some regions, a certain amount of energy caused clear-cut RPE damage; in adjacent regions, the same energy caused little or no RPE cell damage (e.g., Figure 8b,c lesions applied with $44 \mu \mathrm{J}$ $397 \mathrm{~mJ} / \mathrm{cm}^{2}$ and pulses of $10 \mu \mathrm{s}$ duration). This variability is important with regard to possible over- and under-treatment of patients. In the absence of laser dosimetry control (or a laser treatment system with marked inhomogeneity due to a high IMF or strongly varying laser pulse energy), overtreatment may occur even with pulse durations that are considered as selective. Our findings demonstrate that OCT-based dosimetry can prevent overtreatment and collateral retinal damage. 


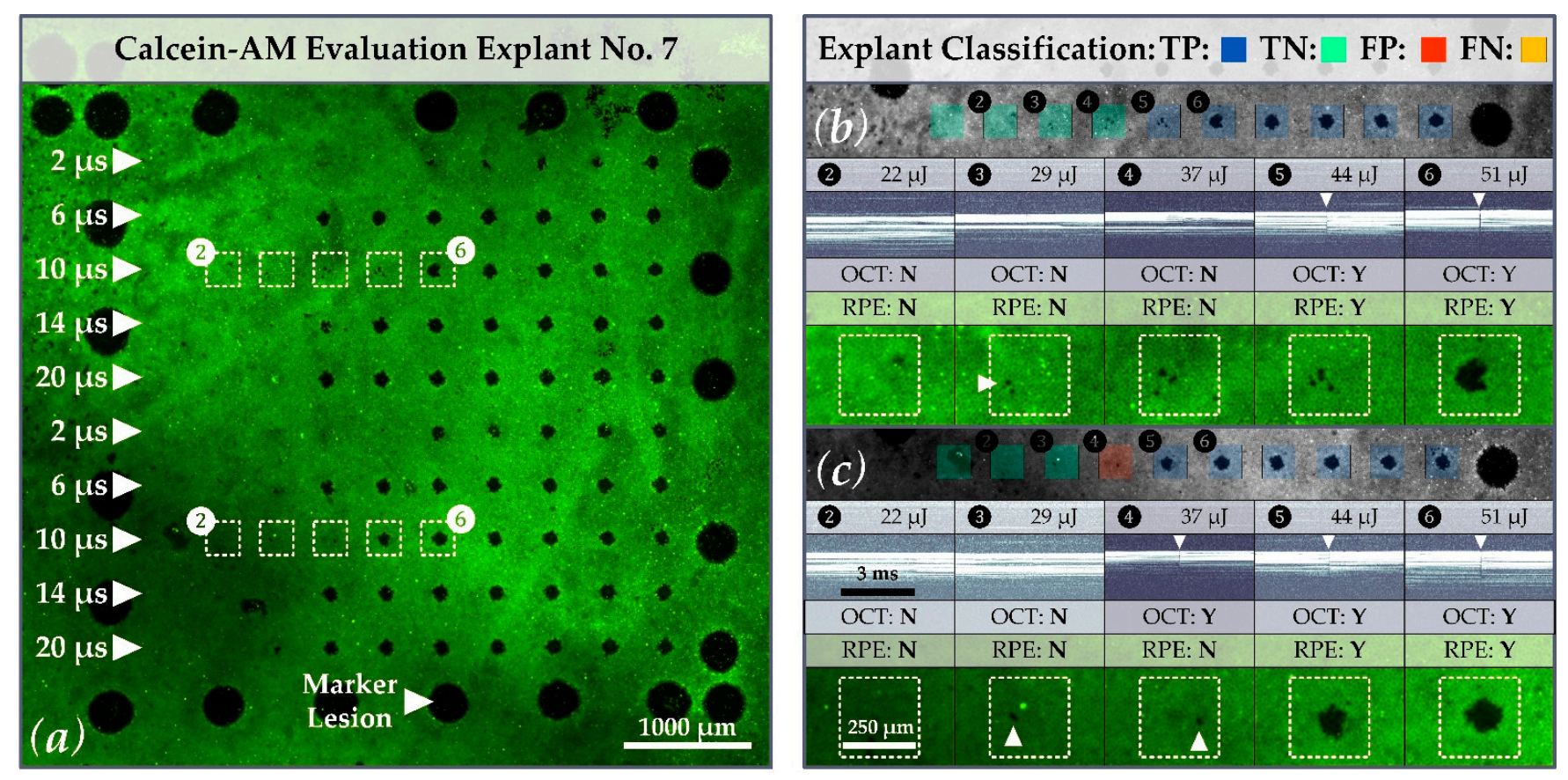

Figure 8. (a) Calcein-AM image of porcine RPE-choroid-sclera explant No. 7. Focus is on the treatment lesions in lines three and eight which were applied with laser pulses of $10 \mu$ s duration (white rectangles with lesions 2 to 6 (white-dashed)). When comparing sections (b) and (c), variations for the threshold of RPE cell damage can be observed. Whereas for treatment line three, a distinctive lesion can be observed at a pulse energy of $51 \mu \mathrm{J} 460 \mathrm{~mJ} / \mathrm{cm}^{2}$, a similar distinctive lesion for treatment line eight is already present at a pulse energy of $44 \mu \mathrm{J} 397 \mathrm{~mJ} / \mathrm{cm}^{2}$. Furthermore, for each lesion in sections $(\mathbf{b}, \mathbf{c})$, the corresponding SD-OCT M-scan, as well as an explant classification according to the confusion matrix, is presented. Local RPE cell destructions that may be related to intraindividual melanin concentrations or local intensity peaks in the beam profile are marked with white triangles (sample classification with a $\kappa$-value of 13 ). The algorithm is partially able to detect MBF through a few damaged RPE cells (e.g., lesion four (c)).

Regarding the IMF, local RPE cell destruction (e.g., lesion $3\left(262 \mathrm{~mJ} / \mathrm{cm}^{2} \hat{=} 29 \mu \mathrm{J}\right)$ in Figure $8 \mathrm{~b}$ ) may be attributed to local peaks in the laser beam profile, in addition to local pigment differences. For laser treatments that are intended to selectively damage the RPE, the influence of the top-hat beam quality is of fundamental importance. Whereas local intensity peaks can lead to local over-treatment and mechanical rupture, local dips might not lead to any damage at all. In terms of clinical application, the influence of the IMF in this respect is interesting to discuss, because in our experiments the laser was applied to the uncovered RPE situated within an artificial eye with an optically "perfect" cornea and lens. When applied to patients, however, ocular aberrations, cloudiness of the ocular media, and other effects need to be considered. The beam profile of the SRT laser is thus (additionally) reshaped on its way to the RPE, which can strengthen or weaken intensity peaks. In addition, the laser can undergo scattering caused by edema, which may smooth out local intensity peaks. It is therefore difficult to precisely define what IMF value is reasonable, although homogenous exposure $(\mathrm{IMF}=1)$ that excludes ocular influences is desirable.

The correlation between the exposure threshold values for the OCT signal loss in M-scans and RPE cell damage supports the ability of the Spectralis Centaurus system for predictive microsecond laser dosimetry via OCT fringe washout analysis. A similar average threshold for fringe washouts in M-scans would also allow the evaluation of sequential events (e.g., three consecutive fringe washouts) as proposed by Kaufmann et al. (SRT in ramp mode combined with a fast algorithm-based SD-OCT treatment stop). This would make the SRT dosing more robust, without risking over-treatment despite the tissue's variable exposure thresholds [28]. This methodology will be investigated in further experiments with the Spectralis Centaurus system. 
A remaining open question is whether the OCT signal loss is caused solely by MBF or whether pure thermal effects, like fast tissue expansion or coagulation, are also detected. Recently, Seifert et al. concluded that thermal cell damage is likely to be the primary cellular damage mechanism. According to this work, MBF sets in at slightly higher radiant exposure levels and leads to superimposed mechanical damage [40]. Based on ex-vivo experiments, Lee et al. found approximately equal thresholds for MBF and RPE cell damage at $10 \mu \mathrm{s}$, whereas at $20 \mu \mathrm{s}$ the MBF threshold was $~ 10 \%$ higher than for cell damage [18]. Analogous results were recently found by Seifert et al. Their ex vivo results suggested approximately equal thresholds for MBF and RPE cell damage at $2 \mu \mathrm{s}$, whereas for laser pulses of $5.2 \mu$ s and $20 \mu$ s duration, the MBF threshold was $\sim 7 \%$ and $\sim 25 \%$, respectively, which was higher than for cell damage [40]. By contrast, our investigator-based evaluation of OCT M-scans (Appendix A: Figure A1 and Table A2) suggested that OCT signal loss occurs even at exposures $\sim 6 \%$ lower than that at which RPE cell damage occurs, and that this holds true across the whole pulse duration range from 2 to $20 \mu \mathrm{s}$. These results show the exciting potential for OCT-based assessment of RPE cell damage and may have the following causes: (1) MBF is the primary damage mechanism up to $20 \mu \mathrm{s}$. OCT is extremely sensitive to such damage and even detects very small microbubbles that do not actually lead to cell damage. (2) The OCT fringe washout technique is more sensitive for longer pulse durations up to $20 \mu$ s than the optoacoustic and light reflection methods [22,40]. (3) OCT fringe washout detects fast thermal tissue expansion and subsequent thermal cell damage, rather than primarily responding to MBF.

A current weakness of OCT dosimetry in terms of patient application is the limited axial SD-OCT scan depth of $1.8 \mathrm{~mm}$. Therefore, axial movements of the patient as well as drift, tremor and saccades of the eye are a challenge. Such artifacts can lead to falsepositive indications of RPE damage or complete signal loss. Accordingly, we are currently evaluating to what extent eye-tracking and other features of the system (e.g., positioning the patient in a supine position or an optimized energy ramp mode) can be modified to counteract these instabilities. Furthermore, one could also take advantage of small axial SD-OCT scan depth by checking the focal plane of the treatment laser via this measuring range to thereby further increase the safety of the SRT delivery.

The OCT dosing control algorithm was developed for a treatment device with a different positive detection criterion (at least 50\% dead RPE cells within the exposure spot, not a cluster of three dead RPE cells) [37]. This criterion leads to fine fringe washouts being discarded by the algorithm, which results in a low number of falsely identified events (overall FP $=24$ ). Since the algorithm focuses on detecting large RPE lesions, many small lesions were missed, leading to a higher number of missed events (overall FN $=93$ ). The overall moderate sensitivity of $85 \%$ and high specificity of $93 \%$ of the algorithmic evaluation can therefore be attributed to the assumption made during the algorithm development. Therefore, the dosing algorithm for fringe washouts in SD-OCT M-scans should be compared again to RPE damage using a calcein-AM viability assay and an area-based detection criterion (i.e., at least 50\% dead RPE cells). Furthermore, the lesions should be applied with a laser burst or ramp application, as currently advocated for SRT [41]. For clinical application, the OCT algorithm should also be compared to RPE barrier leakage found via fluorescence angiography.

The above discussion shows that the decision regarding whether a lesion is considered successful or unsuccessful can have a major influence on the assessment of a dosimeter's performance. The question therefore arises, of what proportion of the RPE in the area of the applied laser beam needs to be injured for a treatment to be considered successful. It is questionable whether lesioning a cluster of three RPE cells is sufficient to rejuvenate the RPE and stimulate their metabolism sufficiently to have a positive effect on retinal disease. To our knowledge, no in vivo study with patients has gauged the minimum number of lesioned RPE cells necessary for a successful treatment. However, experimental results for ex vivo porcine RPE-choroid explants revealed increased pigment epithelium derived factor (PEDF) secretion in $200 \mu \mathrm{m}$ SRT spots compared to $100 \mu \mathrm{m}$ spots [11]. This indicates 
that laser spot size in SRT plays an important role. Therefore, an area-based classification should be included in future evaluations of SRT dosimetry algorithms. With respect to our OCT dosimetry algorithm, this would probably lead to a higher sensitivity, as fewer false-negative events would be observed. Furthermore, an OCT device that evaluates the emanating pressure field might become more sensitive with either a larger or also smaller detection area, although vibrations obviously span more than the detection range of the $\sim 250 \mu \mathrm{m}$ thick retina.

Our results suggest that RPE cell damage could be successfully monitored by using an OCT dosimetry algorithm. Optimal performance of the algorithm was obtained for a pulse duration of 6 or $14 \mu$ s (AUC 0.97). The OOP was determined for a $\kappa$-value of 13 at a laser pulse duration of $6 \mu$ s (sensitivity: 0.89, specificity: 0.94). However, in the case of OCT dosimetry, sensitivity is of paramount importance because sensitivity is a measure of how many of the positive samples (RPE lesion created) have been predicted correctly by the dosimetry algorithm. Showing a high sensitivity in this case means that lesions within the RPE are detected reliably, such that further (unnecessary) laser pulses are not delivered. For successful SRT operation, overtreatment due to low-sensitivity dosimetry must be avoided. Therefore, the OOP could be biased in favor of sensitivity, which would lead to the use of lower $\kappa$-values (e.g., 12 to 10). When interpreting our results, consideration should be given to the fact that only the RPE, choroid and sclera were present during the SD-OCT M-scan acquisition. Thus, the algorithm had relatively little signal available for the fringe washout evaluation. Similar experiments should therefore be repeated on whole eyes, where the additional OCT signal from the neural retina would likely enhance the MBF detection. Furthermore, since the present results are based on a single pulse application, further studies with pulse sequences should be performed to verify the performance under repeated exposure.

\section{Conclusions}

Our experiments show that OCT dosimetry can reliably monitor sub $20 \mu$ s laser pulse-induced RPE cell damage via automated detection of fringe-washouts. Furthermore, we have demonstrated that SD-OCT, even at integration times much higher than the corresponding expansion time of microbubbles, is sensitive enough to detect the optical signatures of MBF. Indeed, damage was detectable even at low radiant exposure levels, when only a few RPE cells within the treatment spot were ruptured. Future research with this system will need to verify whether real-time OCT dosimetry in patients undergoing SRT is capable of automatically controlling the level of treatment; enough to trigger RPE regeneration but without adverse effects to neighboring tissue. Real-time control holds the promise for significantly reducing the risks of SRT, while also permitting more rapid retinal microsurgery of large areas. While the focus of the current study was specific to RPE-rejuvenation, in principle, OCT-guided control of optical treatment-based on selective absorption features of the tissue - could be implemented in other biological or technical environments to achieve minimally invasive treatment.

Author Contributions: Conceptualization, C.B., A.H., D.T.-K. and R.B.; methodology, C.B., A.H., D.T.-K. and R.B.; software, L.G., P.A. and S.S.; formal analysis, C.B. and A.H.; investigation, C.B. and A.H.; resources, R.B., D.T.-K. and C.M.; data curation, C.B., A.H. and L.G.; writing-original draft preparation, C.B., A.H., R.B. and B.P.; writing-review and editing, R.B., D.T.-K., B.P., C.M., P.A. and A.E.; visualization, C.B., A.H. and S.S.; funding acquisition, B.P. and C.M. All authors have read and agreed to the published version of the manuscript.

Funding: This research was funded by the Swiss National Science Foundation, grant number 325230141189 and 323523-163306 as well as the Swiss Innovation Agency, grant number KTI 25030.1 PFLS-LS.

Institutional Review Board Statement: Not applicable.

Informed Consent Statement: Not applicable. 
Data Availability Statement: The data presented in this study (excluding the SD-OCT dosimetry algorithm) are available on request from the corresponding author.

Acknowledgments: We gratefully want to acknowledge the valuable support and the inspiring discussions with our colleagues Veit Danicke, Medical Laser Center Lübeck; Martin Frenz, Institute of Applied Physics of the University of Bern; Jeremy Guggenheim, University of Cardiff; Markus Stoller, Michael Stetter and Eric Odenheimer, Meridian AG; Ralf Kessler, Stefan Schmidt, Michael Reutter, Joerg Fischer and Tilman Otto, Heidelberg Engineering.

Conflicts of Interest: The authors declare no conflict of interest.

\section{Appendix A}

The exact radiant exposure values per lesion for the probe region are provide in Table A1. In addition to the OCT M-scan evaluation using the dosimetry algorithm, an investigator-based evaluation of the M-scan evaluation is provided as well. The binary evaluation and comparison with RPE cell damage thresholds are represented like Figure 6 by using a Probit-Plot.

\section{Appendix A.1. Treatment Pattern and Irradiation}

As depicted in Figure 2a, the pulse duration was increased from top to bottom ( 2 to $20 \mu \mathrm{s})$, and the radiant exposure was increased from left $(135 \mathrm{~mJ} / \mathrm{cm} 2 \hat{=} 15 \mu \mathrm{J})$ to right (maximum: $1354 \mathrm{~mJ} / \mathrm{cm} 2 \hat{=} 150 \mu \mathrm{J}$ at $20 \mu \mathrm{s}$ ). The exact radiant exposure values per lesion can be found in Table A1.

Table A1. Laser treatment pattern for microsecond single pulse laser exposure: applied pulse duration ( $\mu$ s) with the corresponding target energy $(\mu \mathrm{J})$ and radiant exposure $\left(\mathrm{mJ} / \mathrm{cm}^{2}\right)$ per lesion number (1 to $10 /$ from left to right).

\begin{tabular}{|c|c|c|c|c|c|c|c|c|c|c|c|}
\hline \multirow{2}{*}{ Pulse Duration } & \multicolumn{10}{|c|}{ Target Energy and Corresponding Radiant Exposure ${ }^{1}$ per Lesion } & \\
\hline & 1 & 2 & 3 & 4 & 5 & 6 & 7 & 8 & 9 & 10 & \\
\hline \multirow{2}{*}{$2 \mu \mathrm{s}$} & 15 & 17 & 19 & 21 & 23 & 26 & 28 & 30 & 32 & 34 & $\mu \mathrm{J}$ \\
\hline & 135 & 153 & 172 & 190 & 208 & 235 & 253 & 271 & 289 & 307 & $\mathrm{~mJ} / \mathrm{cm}^{2}$ \\
\hline \multirow{2}{*}{$6 \mu \mathrm{s}$} & 15 & 22 & 29 & 37 & 44 & 51 & 58 & 66 & 73 & 80 & $\mu \mathrm{J}$ \\
\hline & 135 & 199 & 262 & 334 & 397 & 460 & 524 & 596 & 659 & 722 & $\mathrm{~mJ} / \mathrm{cm}^{2}$ \\
\hline \multirow{2}{*}{$10 \mu \mathrm{s}$} & 15 & 22 & 29 & 37 & 44 & 51 & 58 & 66 & 73 & 80 & $\mu \mathrm{J}$ \\
\hline & 135 & 199 & 262 & 334 & 397 & 460 & 524 & 596 & 659 & 722 & $\mathrm{~mJ} / \mathrm{cm}^{2}$ \\
\hline \multirow{2}{*}{$14 \mu \mathrm{s}$} & 15 & 28 & 41 & 53 & 66 & 79 & 92 & 104 & 117 & 130 & $\mu J$ \\
\hline & 135 & 253 & 370 & 478 & 596 & 713 & 831 & 939 & 1056 & 1174 & $\mathrm{~mJ} / \mathrm{cm}^{2}$ \\
\hline \multirow{2}{*}{$20 \mu \mathrm{s}$} & 15 & 30 & 45 & 60 & 75 & 90 & 105 & 120 & 135 & 150 & $\mu \mathrm{J}$ \\
\hline & 135 & 271 & 406 & 542 & 677 & 813 & 948 & 1083 & 1219 & 1354 & $\mathrm{~mJ} / \mathrm{cm}^{2}$ \\
\hline
\end{tabular}

${ }^{1}$ According to Equation (1): spot size on explant $\mathrm{A}=120 \times 120 \mu \mathrm{m}^{2} ; \mathrm{IMF}=1.3$.

Appendix A.2. RPE-Cell Damage and M-Scan Signal Washout Thresholds for Single Pulse Irradiation

Table A2 shows the calculated radiant exposure thresholds for RPE-cell damage and M-scan fringe washouts by single irradiation for different pulse durations.

In addition to the OCT data evaluation by the dosimetry algorithm, an investigatorbased visual evaluation was performed. In the investigator-based evaluation, the M-scan evaluation took place post-treatment by reviewing image by image for every treatment point. For the evaluation, a visible washout in the M-scan was rated with 1 and in case of absence with 0 . A fringe-washout was only scored if all structures in the SD-OCT M-scan were affected (completely throughout signal loss). The threshold for the investigator-based evaluation is shown in the Probit-Plot in Figure A1b. 

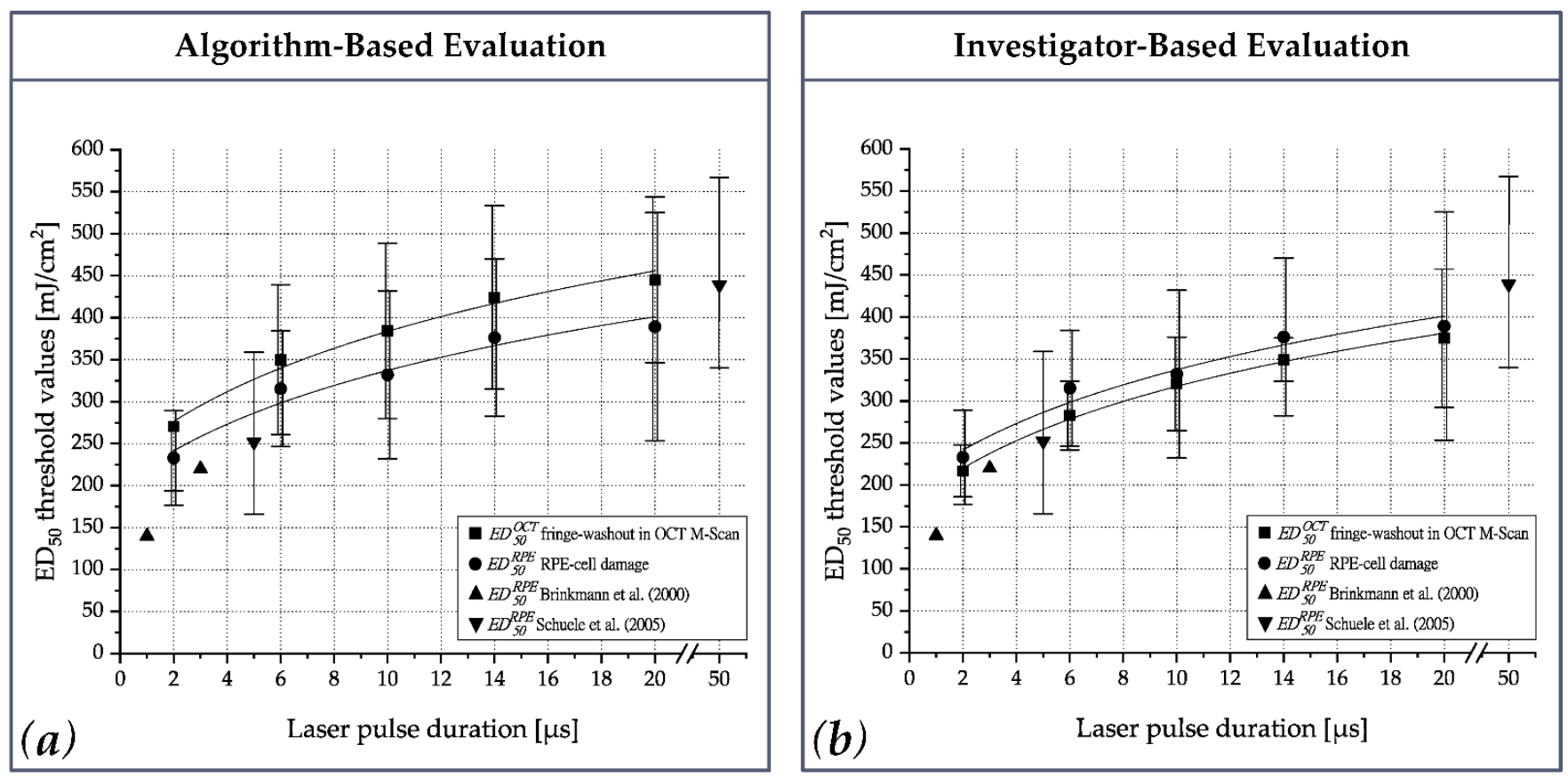

Figure A1. $E D_{50}$ thresholds values $(\mathrm{mJ} / \mathrm{cm} 2)$ for RPE-cell damage $\left(E D_{50}^{R P E}\right)$ and fringe washouts in OCT M-scans $\left(E D_{50}^{O C T}\right)$ for single pulse laser irradiation $(2 \mu \mathrm{s}, 6 \mu \mathrm{s}, 10 \mu \mathrm{s}, 14 \mu \mathrm{s}$ and $20 \mu \mathrm{s})$; (a) OCT dosimetry algorithm-based evaluation (corresponds to Figure 5); (b) Investigator-based evaluation.

Table A2. Threshold values for RPE-cell damage and fringe washouts in OCT M-scans (algorithmic analysis) for microsecond single pulse laser irradiation.

\begin{tabular}{|c|c|c|c|c|c|c|c|c|c|c|}
\hline \multirow{3}{*}{ Pulse Duration } & \multicolumn{9}{|c|}{ Energy and Corresponding Radiant Exposure ${ }^{1}$ Threshold Values } & \\
\hline & \multirow{2}{*}{$E D_{15}^{R P E}$} & \multirow{2}{*}{$E D_{50}^{R P E}$} & \multirow{2}{*}{$E D_{85}^{R P E}$} & \multicolumn{3}{|c|}{ Algorithm-Based } & \multicolumn{3}{|c|}{ Investigator-Based } & \\
\hline & & & & $E D_{15}^{O C T}$ & $E D_{50}^{O C T}$ & $E D_{85}^{O C T}$ & $E D_{15}^{O C T}$ & $E D_{50}^{O C T}$ & $E D_{85}^{O C T}$ & \\
\hline \multirow{2}{*}{$2 \mu \mathrm{s}$} & 25 & 34 & 42 & 28 & 39 & - & 27 & 31 & 36 & $\mu \mathrm{J}$ \\
\hline & 177 & 233 & 289 & 194 & 270 & - & 186 & 217 & 248 & $\mathrm{~mJ} / \mathrm{cm}^{2}$ \\
\hline \multirow{2}{*}{$6 \mu \mathrm{s}$} & 36 & 45 & 55 & 38 & 50 & 63 & 35 & 41 & 47 & $\mu \mathrm{J}$ \\
\hline & 247 & 315 & 384 & 260 & 350 & 439 & 242 & 283 & 324 & $\mathrm{~mJ} / \mathrm{cm}^{2}$ \\
\hline \multirow{2}{*}{$10 \mu \mathrm{s}$} & 33 & 48 & 62 & 40 & 55 & 70 & 38 & 46 & 54 & $\mu \mathrm{J}$ \\
\hline & 232 & 332 & 432 & 280 & 384 & 489 & 265 & 320 & 376 & $\mathrm{~mJ} / \mathrm{cm}^{2}$ \\
\hline \multirow{2}{*}{$14 \mu \mathrm{s}$} & 41 & 54 & 68 & 45 & 61 & 77 & 47 & 39 & 54 & $\mu \mathrm{J}$ \\
\hline & 283 & 376 & 470 & 315 & 424 & 533 & 323 & 349 & 375 & $\mathrm{~mJ} / \mathrm{cm}^{2}$ \\
\hline \multirow{2}{*}{$20 \mu \mathrm{s}$} & 37 & 56 & 76 & 50 & 64 & 78 & 42 & 42 & 66 & $\mu \mathrm{J}$ \\
\hline & 253 & 389 & 525 & 346 & 445 & 544 & 292 & 375 & 457 & $\mathrm{~mJ} / \mathrm{cm}^{2}$ \\
\hline
\end{tabular}

${ }^{1}$ According to Equation (1): spot size on explant $\mathrm{A}=120 \times 120 \mu \mathrm{m}^{2} ; \mathrm{IMF}=1.3$.

\section{References}

1. Meyer-Schwickerath, G. Lichtkoagulation; eine Methode zur Behandlung und Verhütung der Netzhautablösung. Graefe's Arch. Clin. Exp. Ophthalmol. 1954, 156, 2-34. [CrossRef]

2. Early Treatment Diabetic Retinopathy Study Research Group. Early photocoagulation for diabetic retinopathy: ETDRS report number 9. Ophthalmology 1991, 98, 766-785. [CrossRef]

3. Early Treatment Diabetic Retinopathy Study Research Group. Photocoagulation for diabetic macular edema. Arch. Ophthalmol. 1985, 103, 1796-1806. [CrossRef]

4. Nicholson, B.; Noble, J.; Forooghian, F.; Meyerle, C. Central Serous Chorioretinopathy: Update on Pathophysiology and Treatment. Surv. Ophthalmol. 2013, 58, 103-126. [CrossRef] [PubMed]

5. Framme, C.; Schuele, G.; Roider, J.; Birngruber, R.; Brinkmann, R. Influence of pulse duration and pulse number in selective RPE laser treatment. Lasers Surg. Med. 2004, 34, 206-215. [CrossRef] 
6. Kozak, I.; Luttrull, J.K. Modern retinal laser therapy. Saudi J. Ophthalmol. 2015, 29, 137-146. [CrossRef] [PubMed]

7. Birngruber, R.; Gabel, V.P.; Hillenkamp, F. Experimental studies of laser thermal retinal injury. Health Phys. 1983, 44, 519-531. [CrossRef] [PubMed]

8. Birngruber, R.; Hillenkamp, F.; Gabel, V.P. Theoretical investigations of laser thermal retinal injury. Health Phys. 1985, $48,781-796$. [CrossRef] [PubMed]

9. Brinkmann, R.; Roider, J.; Birngruber, R. Selective retina therapy (SRT): A review on methods, techniques, preclinical and first clinical results. Bull. Soc. Belge Ophtalmol 2006, 302, 51-69.

10. Roider, J.; Michaud, N.A.; Flotte, T.J.; Birngruber, R. Response of the retinal pigment epithelium to selective photocoagulation. Arch. Ophthalmol. 1992, 110, 1786-1792. [CrossRef]

11. Richert, E.; Koinzer, S.; Tode, J.; Schlott, K.; Brinkmann, R.; Hillenkamp, J.; Klettner, A.; Roider, J. Release of Different Cell Mediators During Retinal Pigment Epithelium Regeneration Following Selective Retina Therapy. Investig. Ophthalmol. Vis. Sci. 2018, 59, 1323-1331. [CrossRef] [PubMed]

12. Kang, S.; Park, Y.G.; Kim, J.R.; Seifert, E.; Theisen-Kunde, D.; Brinkmann, R.; Dirk, T.K.; Ralf, B.; Roh, Y.J. Selective Retina Therapy in Patients With Chronic Central Serous Chorioretinopathy: A Pilot Study. Medicine 2016, 95. [CrossRef] [PubMed]

13. Gabel, V.P.; Birngruber, R.; Hillenkamp, F. Visible and near infrared light absorption in pigment epithelium and choroid. XXIII Concil. Ophthaimologicum 1978, 450, 658-662.

14. Neumann, J.; Brinkmann, R. Boiling nucleation on melanosomes and microbeads transiently heated by nanosecond and microsecond laser pulses. J. Biomed. Opt. 2005, 10, 024001. [CrossRef]

15. Neumann, J.; Brinkmann, R. Cell disintegration by laser-induced transient microbubbles and its simultaneous monitoring by interferometry. J. Biomed. Opt. 2006, 11, 041112. [CrossRef]

16. Roider, J.; Hillenkamp, F.; Flotte, T.; Birngruber, R. Microphotocoagulation: Selective effects of repetitive short laser pulses. Proc. Natl. Acad. Sci. USA 1993, 90, 8643-8647. [CrossRef] [PubMed]

17. Schuele, G.; Elsner, H.; Framme, C.; Roider, J.; Birngruber, R.; Brinkmann, R. Optoacoustic real-time dosimetry for selective retina treatment. J. Biomed. Opt. 2005, 10, 064022. [CrossRef]

18. Lee, H.; Alt, C.; Pitsillides, C.; Lin, C. Optical detection of intracellular cavitation during selective laser targeting of the retinal pigment epithelium: Dependence of cell death mechanism on pulse duration. J. Biomed. Opt. 2007, 12, 064034. [CrossRef]

19. Roider, J.; Lindemann, C.; El-Hifnawi, E.S.; Laqua, H.; Birngruber, R. Therapeutic range of repetitive nanosecond laser exposures in selective RPE photocoagulation. Graefe's Arch. Clin. Exp. Ophthalmol. 1998, 236, 213-219. [CrossRef]

20. Framme, C.; Schuele, G.; Kobuch, K.; Flucke, B.; Birngruber, R.; Brinkmann, R. Investigation of selective retina treatment (SRT) by means of $8 \mathrm{~ns}$ laser pulses in a rabbit model. Lasers Surg. Med. 2008, 40, 20-27. [CrossRef] [PubMed]

21. Roider, J.; Liew, S.H.; Klatt, C.; Elsner, H.; Poerksen, E.; Hillenkamp, J.; Brinkmann, R.; Birngruber, R. Selective retina therapy (SRT) for clinically significant diabetic macular edema. Graefe's Arch. Clin. Exp. Ophthalmol. 2010, 248, 1263-1272. [CrossRef] [PubMed]

22. Seifert, E.; Tode, J.; Pielen, A.; Theisen-Kunde, D.; Framme, C.; Roider, J.; Miura, Y.; Birngruber, R.; Brinkmann, R. Selective retina therapy: Toward an optically controlled automatic dosing. J. Biomed. Opt. 2018, 23, 115002. [CrossRef] [PubMed]

23. Seifert, E.; Roh, Y.-J.; Fritz, A.; Park, Y.G.; Kang, S.; Theisen-Kunde, D.; Brinkmann, R. Automatic irradiation control by an optical feedback technique for selective retina treatment (SRT) in a rabbit model. In Proceedings of the Medical Laser Applications and Laser-Tissue Interactions VI, Munich, Germany, 12-16 May 2013.

24. Steiner, P.; Enzmann, V.; Meier, C.; Považay, B.; Kowal, J.H. Retinal Laser Lesion Visibility in Simultaneous Ultra-High Axial Resolution Optical Coherence Tomography. IEEE Photon. J. 2014, 6, 1-11. [CrossRef]

25. Steiner, P.; Ebneter, A.; Berger, L.E.; Zinkernagel, M.; Považay, B.; Meier, C.; Kowal, J.H.; Framme, C.; Brinkmann, R.; Wolf, S.; et al. Time-Resolved Ultra-High Resolution Optical Coherence Tomography for Real-Time Monitoring of Selective Retina Therapy. Investig. Ophthalmol. Vis. Sci. 2015, 56, 6654-6662. [CrossRef]

26. Steiner, P.; Považay, B.; Stoller, M.; Morgenthaler, P.; Inniger, D.; Arnold, P.; Sznitman, R.; Meier, C. Real-time optical coherence tomography observation of retinal tissue damage during laser photocoagulation therapy on ex-vivo porcine samples. In Proceedings of the Optical Coherence Imaging Techniques and Imaging in Scattering Media, Munich, Germany, 21-25 June 2015.

27. Kaufmann, D.; Burri, C.; Arnold, P.; Koch, V.M.; Meier, C.; Považay, B.; Justiz, J. Dosimetry Control and Monitoring of Selective Retina Therapy using Optical Coherence Tomography. In Proceedings of the Optical Coherence Imaging Techniques and Imaging in Scattering Media II, Munich, Germany, 25-29 June 2017.

28. Kaufmann, D.; Burri, C.; Arnold, P.; Koch, V.M.; Meier, C.; Považay, B.; Justiz, J. Selective retina therapy enhanced with optical coherence tomography for dosimetry control and monitoring: A proof of concept stud. Biomed. Opt. Express 2018, 9, 3320-3334. [CrossRef]

29. Lee, S.; Wei, S.; Guo, S.; Kim, J.; Kim, B.; Kim, G.; Kang, J.U. Selective retina therapy monitoring by speckle variance optical coherence tomography for dosimetry control. J. Biomed. Opt. 2020, 25, 026001. [CrossRef]

30. Považay, B.; Brinkmann, R.; Stoller, M.; Kessler, R. High Resolution Imaging in Microscopy and Ophthalmology—New Frontiers in Biomedical Optics; Bille, J.F., Ed.; Springer: Berlin/Heidelberg, Germany, 2019; pp. 237-259.

31. Framme, C.; Schuele, G.; Roider, J.; Kracht, D.; Birngruber, R.; Brinkmann, R. Threshold determinations for selective retinal pigment epithelium damage with repetitive pulsed microsecond laser systems in rabbits. Ophthalmic Surg. Lasers Imaging Retin. 2002, 33, 400-409. [CrossRef] 
32. Hutfilz, A.; Burri, C.; Theisen-Kunde, D.; Meier, C.; Brinkmann, R. Ex Vivo Investigation of Different Ms Laser Pulse Durations for Selective Retina Therapy. In Proceedings of the Clinical and Preclinical Optical Diagnostics II, Munich, Germany, 23-25 June 2019.

33. Sparrrow, J.R.; Hicks, D.; Hamel, C.P. The Retinal Pigment Epithelium in Health and Disease. Curr. Mol. Med. $2010,10,802-823$. [CrossRef] [PubMed]

34. Miura, Y. Retinal pigment epithelium—choroid organ culture. Expert Rev. Ophthalmol. 2011, 6, 669-680. [CrossRef]

35. Sliney, D.H.; Mellerio, J.; Gabel, V.P.; Schulmeister, K. What Is the Meaning of Threshold in Laser Injury Experiments? Implications for Human Exposure Limits. Health Phys. 2002, 82, 335-347. [CrossRef]

36. Schindelin, J.; Arganda-Carreras, I.; Frise, E.; Kaynig, V.; Longair, M.; Pietzsch, T.; Preibisch, S.; Rueden, C.; Saalfeld, S.; Schmid, B.; et al. Fiji: An open-source platform for biological-image analysis. Nat. Methods 2012, 9, 676-682. [CrossRef] [PubMed]

37. Grimm, L. Optical Coherence Tomography for Real-Time Automatic Dosimetry Control in Selective Retina Therapy. Master's Thesis, University of Bern, Bern, Switzerland, 2019.

38. Brinkmann, R.; Huettmann, G.; Roegener, J.; Roider, J.; Birngruber, R.; Lin, C. Origin of retinal pigment epithelium cell damage by pulsed laser irradiance in the nanosecond to microsecond time regimen. Lasers Surg. Med. 2000, 27, 451-464. [CrossRef]

39. Schuele, G.; Rumohr, M.; Huettmann, G.; Brinkmann, R. RPE damage thresholds and mechanisms for laser exposure in the microsecond-to-millisecond time regimen. Investig. Ophthalmol. Vis. Sci. 2005, 46, 714-719. [CrossRef]

40. Seifert, E.; Sonntag, S.R.; Kleingarn, P.; Theisen-Kunde, D.; Grisanti, S.; Birngruber, R.; Miura, Y.; Brinkmann, R. Investigations on Retinal Pigment Epithelial Damage at Laser Irradiation in the Lower Microsecond Time Regime. Investig. Ophthalmol. Vis. Sci. 2021, 62, 32. [CrossRef] [PubMed]

41. Kim, Y.J.; Lee, Y.G.; Lee, D.W.; Kim, J.H. Selective Retina Therapy with Real-Time Feedback-Controlled Dosimetry for Treating Acute Idiopathic Central Serous Chorioretinopathy in Korean Patients. J. Ophthalmol. 2018, 6027871, 2018. [CrossRef] [PubMed] 